\title{
Developmental Tuning in a Spinal Nociceptive System: Effects of Neonatal Spinalization
}

\author{
Anders Levinsson, Xiao-Ling Luo, Hans Holmberg, and Jens Schouenborg \\ Department of Physiological Sciences, Section for Neurophysiology, Lund University, S-223 62 Lund, Sweden
}

Recent studies indicate a modular organization of the nociceptive withdrawal reflex system. Each module has a characteristic receptive field, closely matching the withdrawal movement caused by its effector muscle. In the rat, the strength of the sensory input to each module is tuned during the first postnatal weeks, i.e., erroneous spinal connections are depressed, and adequate connections are strengthened. To clarify if this tuning is dependent on supraspinal structures, the effect of a complete neonatal spinal cord transection on the postnatal tuning of withdrawal reflexes was studied. The nociceptive receptive fields of single hindlimb muscles and compound withdrawal reflexes were examined in decerebrate unanesthetized and awake rats, respectively. Noxious thermal $\mathrm{CO}_{2}$ laser stimulation was used to evoke reflex responses. Neonatal spinal cord transection resulted in a disrupted reflex organization in the adult rat, resembling that previously found in neonatal rats. The receptive fields of single hindlimb muscles exhibited abnormal distribution of sensitivity not matching the withdrawal action of the effector muscles. Likewise, the composite nocifensive movements, as documented in the awake rat, often resulted in erroneous movements toward the stimulus. It is concluded that withdrawal reflexes do not become functionally adapted in rats spinalized at birth. These findings suggest a critical role for supraspinal systems in the postnatal tuning of spinal nociceptive systems.

Key words: pain; plasticity; sensorimotor integration; spasticity; brainstem control; rat
Motor systems typically receive considerable somatosensory input (Porter and Lemon, 1993). To be useful in sensorimotor transformations, the neural connections mediating the sensory input and their "weight" must be tuned to the function of the motor system. Information on how such connections are tuned would help understand the functional organization of the adult motor systems and may also shed light on the plastic changes in motor systems after injury and reconstructive surgery.

One system suitable for studies of how sensorimotor transformations are functionally tuned is the nociceptive withdrawal reflex (NWR) system. Recent studies indicate that this spinal system has a "modular" organization, each module essentially controlling a single muscle (Schouenborg and Kalliomäki, 1990; Schouenborg et al., 1992, 1994; Schouenborg and Weng, 1994; Weng and Schouenborg, 1996a). The receptive field of a module is highly characteristic. Its location and the distribution of sensitivity within the field closely mirrors the withdrawal movement pattern of the skin surface produced by the effector muscle or muscles when the animal is in the standing position. Thus, the weight of the sensory input to a given module is tuned to match the output, i.e., withdrawal of the skin.

In neonatal rats, NWRs evoked by cutaneous stimulation are

\footnotetext{
Received May 7, 1999; revised Aug. 13, 1999; accepted Sept. 10, 1999.

This work was supported by the Swedish Medical Research Council Projects No. 10569 and 1013, the Medical Faculty of Lund University, Astra Hässle, Inc., Knut and Alice Wallenberg's Foundation, Elsa and Thorsten Segerfalk's Foundation, Greta and Johan Kock's Foundation, the Crafoordska Foundation, and The Royal Physiographic Society in Lund.

Correspondence should be addressed to Anders Levinsson, Department of Physiological Sciences, Section for Neurophysiology, Lund University, Sölvegatan 19, S-22362 Lund, Sweden. E-mail: Anders.Levinsson@mphy.lu.se.

Dr. Luo's present address: Department of Neuroscience, Pathology building 2-210, Johns Hopkins University School of Medicine, 600 North Wolfe Street, Baltimore, MD 21287.

Copyright (C) 1999 Society for Neuroscience $\quad 0270-6474 / 99 / 1910397-07 \$ 05.00 / 0$
}

not functionally adapted, often leading to movements directed toward the stimulation (Holmberg and Schouenborg, 1996a). Then, the adult task-specific NWR organization gradually emerges over the first three postnatal weeks. During this process, erroneous connections are depressed, and adequate connections are strengthened. The system can adapt to both altered sensory innervation of the periphery at birth (Holmberg and Schouenborg, 1996b) and to changes of movement pattern caused by neonatal tendon transfer (Holmberg et al., 1997). We have suggested that the cutaneous sensory feedback ensuing on contraction of single muscles is instrumental in the postnatal tuning of the afferent connections to the modules (Schouenborg and Weng, 1994; Holmberg and Schouenborg, 1996a,b; Holmberg et al., 1997).

It is well known that the spinal reflex circuits are normally subject to considerable supraspinal control. The descending systems arise primarily from the brainstem and cerebral cortex and use several different neurotransmitters (Willis and Westlund, 1997). In the rat, most of the tracts projecting to the spinal cord seem to be established within the first postnatal weeks (Leong et al., 1984; Kudo et al., 1993; Porter and Lemon, 1993). It is therefore possible that the postnatal tuning of withdrawal reflex pathways is dependent on supraspinal systems. To address this issue, we have investigated the nociceptive withdrawal reflex system in adult rats subjected to spinal transection at the thoracic level at birth.

Parts of this paper have been published previously (Schouenborg et al., 1996).

\section{MATERIALS AND METHODS}

The present report is based on data from a total of 35 Wistar rats of both sexes. Of these, 22 served as controls and have been included in previous studies (Holmberg and Schouenborg, 1996a,b). The animals received food and water ad libitum and were kept in a $12 \mathrm{hr}$ light/dark cycle at a 


\section{Normal intact adult Chronic spinal adult}
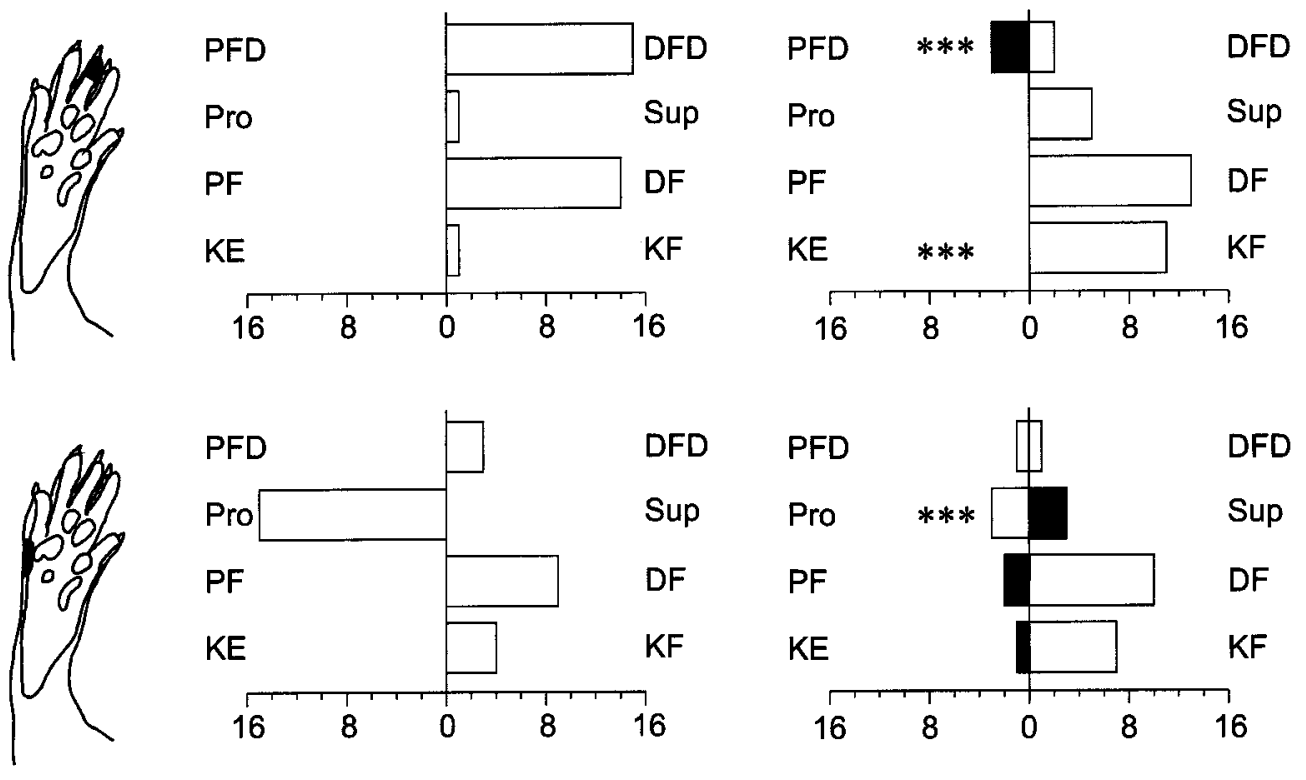

Figure 1. $\mathrm{CO}_{2}$ laser-evoked reflex movements of the right hindlimb. Three different sites on the hindpaw (indicated in each row) were stimulated in awake rats. Each site was stimulated twice. Results from eight rats spinalized at birth (right column) and eight normal rats (left column) are shown. Stimulation intensity was twice the reflex threshold. Each bar represents the total number of movements. Filled bars represent erroneous movements, i.e., those directed toward the stimulation. Statistics refer to comparison with corresponding responses in normal adult rats: ${ }^{*} p<0.05 ;{ }^{* *} p<$ $0.01 ;{ }^{* *} p<0.001$. PFD, Plantar flexion of digits; $D F D$, dorsiflexion of digits; Pro, pronation; Sup, supination; $D F$, dorsiflexion of ankle; $P F$, plantar flexion of ankle; $K E$, knee extension; $K F$, knee flexion.
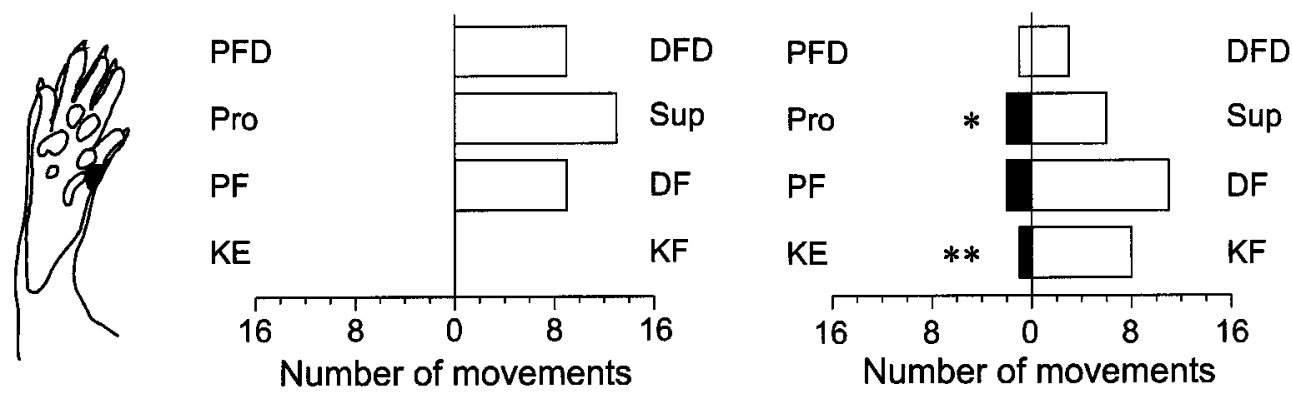

constant environmental temperature of $21^{\circ} \mathrm{C}$ (humidity 65\%). Approval for the experiments was obtained in advance from the Local Ethical Committee of Lund University.

Neonatal surgery. The rats were removed from the home cage on the day of birth and anesthetized by hypothermia (cooling on ice). Three or four pups from each litter were used. Careful infiltration with $2.0 \mathrm{mg} / \mathrm{ml}$ lignocaine (Xylocaine) with $1.2 \mu \mathrm{g} / \mathrm{ml}$ adrenaline was performed before surgery to reduce possible nociceptive input and to minimize bleeding. Surgery commenced when spontaneous movements had ceased and when no reflexes could be evoked by pinching the skin.

A small laminectomy was made, and the spinal cord was transected at the level of T8-11. Skin incisions were closed using fine resorbable thread (Vicryl, 8-0) and Nobecutan (Astra Tech Inc., Mölndal, Sweden) wound spray. The rats were then allowed to recover in a temperaturecontrolled environment and were returned to the home cage after regaining normal skin temperature (measured by a noncontact infrared detector probe; Thermonitor C-1600M; Linear Laboratories, Los Altos, $\mathrm{CA})$. The rats included in the study recovered uneventfully without exhibiting any signs of suffering after surgery. There were no signs of infection during subsequent growth (monitored daily until the day of the acute experiment). Bladder evacuation was not necessary, and there was no marked atrophy of the hindlimb muscles. The general condition of the rats, with the exception of those discarded (see below), appeared excellent as judged from, e.g., condition of fur and level of general activity. Five other rats were discarded from the study because of signs of urinary tract infections.

Studies on nocifensive behavior in awake adult rats. Compound withdrawal reflex patterns were studied in eight chronic spinal rats and eight normal awake rats, aged 10-16 weeks. The rats were handled by the experienced staff of the animal house until they rested quietly. They did not struggle or show any other signs of discomfort during the experiment. The baseline temperature of the hindpaw and tail skin $\left(29-33^{\circ} \mathrm{C}\right)$ was measured by a noncontact probe (see above). The threshold intensity for evoking a visible reflex response using $\mathrm{CO}_{2}$ laser stimulation (Directed Energy Inc., Irvine, CA; unfocused beam, diameter $1.1 \mathrm{~mm}$, intensity 1 $\mathrm{W}$, pulse duration $5-30 \mathrm{msec}$ ) was measured before documentation of reflex responses. Reflexes elicited by $\mathrm{CO}_{2}$ laser pulses at an intensity of twice the reflex threshold were recorded on videotape. When tested on the dorsal side of the experimenters' hands, the stimulation intensities used elicited a weak and very short-lasting pricking pain. When stimulated, the rats exhibited no vocalization or aggressive behavior. No visible skin damage was observed. Three sites, on the hindpaw (Fig. 1) and on the distal and proximal tail bilaterally (within the most distal and proximal $10 \mathrm{~mm}$ segments, respectively), were stimulated twice in each rat. The interstimulus interval was set at $>10 \mathrm{~min}$, thus precluding "wind-up" in nociceptive pathways (Mendell, 1966; Schouenborg, 1984). The videotape was replayed frame by frame (image magnification, $10 \times$; time interval between each frame, $40 \mathrm{msec}$; a light-emitting diode indicated stimulation onset), allowing a qualitative analysis of the initial evoked reflex responses.

Movements of the digits were classified as dorsiflexion (DFD) or plantar flexion (PFD). Ankle movements were classified as supination (Sup) or pronation (Pro) and as dorsiflexion (DF) or plantarflexion (PF). Movements in the knee joint were classified as extension (KE) or flexion (KF). Tail movements were classified as movements toward or away from the stimulus.

Surgery and preparation of adult rats. After the rats had reached adulthood (10-16 weeks, 5 chronic spinal and 14 acute spinal), they were anesthetized with halothane $(1.0-2.0 \%)$ in a mixture of $65 \%$ nitrous oxide and $35 \%$ oxygen and were ventilated artificially via a tracheal cannula. The expiratory $\mathrm{CO}_{2}(3.0-4.5 \%)$ was monitored continuously. An infusion of $5 \%$ glucose in Ringer's solution and acetate, $\mathrm{pH} 7.0$, at a rate of $30-50 \mu \mathrm{l} / \mathrm{min}$, was administered via the right jugular vein. Mean arterial blood pressure $(75-140 \mathrm{mmHg}$ ) was monitored continuously in the right carotid artery. Core temperature was maintained between 36.5 and $38.5^{\circ} \mathrm{C}$ using a thermostatically controlled, feedback-regulated heat- 
ing system. Local infiltration of lignocaine (Xylocaine) with adrenaline (concentrations as above) was used to reduce nociceptive input during surgery and to minimize possible postoperative excitability changes (Clarke and Matthews, 1990). A craniotomy was performed, and the brain rostral to the inferior colliculus was removed by suction. Anesthesia was then discontinued. In the control rats, a laminectomy of the tenth thoracic vertebrae was performed, and the rat was spinalized using a pair of fine scissors. A small incision was made in the skin overlying the muscles under investigation to facilitate insertion of the recording needles into the muscle bellies (see below). Experiments were terminated after signs of deterioration, i.e., a precipitous drop in blood pressure or in the expiratory $\mathrm{CO}_{2}$ level. After termination of the experiments, the animals were given a lethal dose of halothane.

Electromyography recordings. A small opening was made in the skin overlying the muscle belly, and a reference electrode was inserted subcutaneously in an adjacent skin flap. A fine steel needle electrode, which was insulated except for $\sim 50 \mu \mathrm{m}$ at the tip, was inserted into the middle portion of the muscle belly. The identity of the muscle was determined by observing the movements evoked by cathodal pulses $(100 \mathrm{~Hz}, 200 \mathrm{msec}$, $20 \mu \mathrm{A}, 20$ pulses) through the exploring electrode (Schouenborg and Kalliomäki, 1990). The recordings were amplified, filtered (high pass filter, $100 \mathrm{~Hz}$ ), and stored on videotape. Generally, the EMG activity in two or three hindlimb muscles was recorded simultaneously in each experiment.

As judged from the difference in spike amplitudes on noxious stimulation of the skin, multiunit recordings were obtained from each muscle. This is consistent with the fact that any given region in a muscle is shared by many interdigitating motor units (Burke, 1981). No attempt was made to separate the activities of different motor units in the same muscle.

Mapping of cutaneous excitatory receptive fields. Noxious $\mathrm{CO}_{2}$ laser stimulation (parameters as described above) of between 20 and 30 sites on the plantar hindpaw skin was used to map the cutaneous excitatory receptive fields of the extensor digitorum longus muscle (EDL), peroneus longus muscle (PER), and gastrocnemius muscle (GA). To avoid wind-up of responses, the interstimulus interval was $\sim 1 \mathrm{~min}$. Mapping of receptive fields of withdrawal reflexes in control rats started in the interval 4-5 hr after spinalization.

Analysis. The magnitude of the reflex response was defined as the number of motor unit spikes, which could be clearly separated from background noise, evoked during the first second after the onset of the $\mathrm{CO}_{2}$ laser pulse (spike numbers were counted using the EGAA program; RC Electronics, Goleta, CA).

To describe the receptive field of a muscle, the responses in this muscle were plotted on the corresponding stimulation sites on a standard diagram of the hindpaw. Responses were normalized and expressed as the percentage of the maximal response in the respective muscle. They were then transferred mathematically into a high-density grid of evoked responses, which was used to generate an isoresponse map (Kriging algorithm and contour program, Grid and Topo programs; Golden, CO; Schouenborg et al., 1995). Receptive fields were then constructed with the aid of these isoresponse maps and divided into three areas of differing sensitivity: $70-100 \%$ (referred to as receptive field focus), $30-70 \%$, and $0-30 \%$ of maximal response.

Postmortem examination. After the terminal experiments, the spinalized rats were perfused with $10 \%$ formalin in saline. The spinal cords were taken out, and the completeness of the spinalization was assessed.

Statistical analysis. A $\chi^{2}$ test was used for statistical evaluation. Differences were considered statistically significant at the level of $p<0.05$. Each value is given as mean $\pm \mathrm{SD}$.

\section{RESULTS}

Postmortem examinations revealed that, in all but one rat, the spinalizations were complete, often with a gap of several millimeters between the stumps of the cord. In one rat, a very fine thread of white matter remained. The results obtained in this rat did not differ from the rest, and it was therefore included in the analysis (EMG group). Although lacking coordination between forelimbs and hindlimbs, the neonatally spinalized rats could walk and stand on their hindquarters. Typically these rats had somewhat laterally rotated hindpaws and a waddling gait (Stelzner, 1982). These characteristics rendered the chronic spinal rats easily identifiable, and a blinded analysis of behavioral data was therefore not considered feasible.
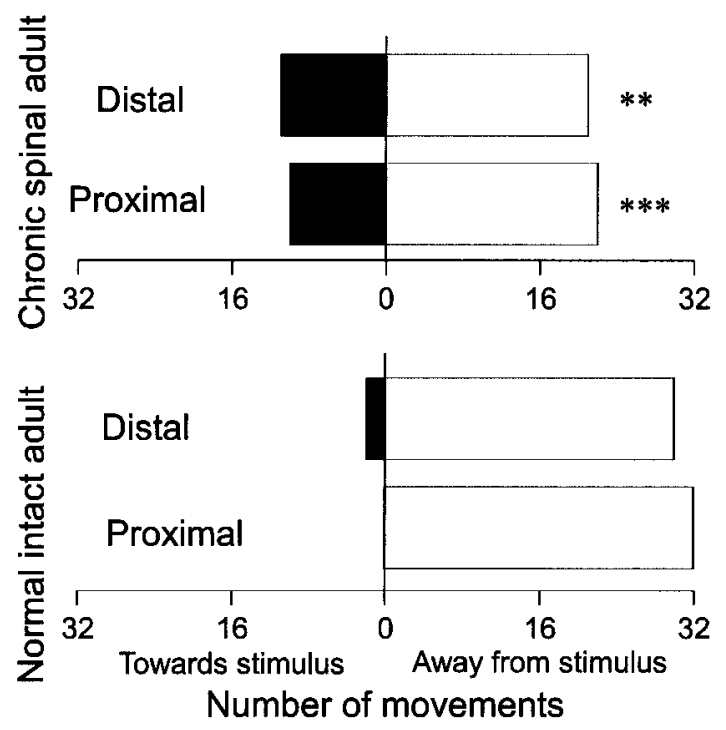

Figure 2. $\mathrm{CO}_{2}$ laser-evoked reflex movements of the tail. The proximal and distal tail was stimulated twice on each side in awake rats. Results from eight rats spinalized at birth (top diagram) and eight normal rats (bottom diagram) are shown. Stimulation intensity was twice the reflex threshold. Filled bars represent erroneous movements, i.e., toward the stimulus. Statistics refer to comparison with normal adult rats: ${ }^{* *} p<0.01$; $* * * p<0.001$.

\section{Behavioral studies}

Withdrawal reflex movements of the hindpaw and tail evoked by $\mathrm{CO}_{2}$ laser stimulation were recorded in the awake chronic spinal rats $(n=8)$, and the spatial patterns were compared with those in normal adult rats $(n=8)$. No clear relation between stimulus site and response pattern could be discerned in the chronic spinal rats. Approximately equal numbers of plantar flexions and dorsiflexions of digits were evoked on stimulation of the plantar side of digit 3 (Fig. 1, top row). Likewise, in the chronic spinal rats, stimulation of the lateral and medial plantar skin did not regularly evoke pronation and supination, respectively (Fig. 1, middle and bottom rows). In contrast, in normal adult rats, stimulation of the plantar side of the digits evokes a dorsiflexion of the digits, and stimulation of the lateral and medial side of the foot evokes pronation and supination, respectively (Fig. 1, left column). Furthermore, proximal hindlimb reflex responses were more frequent in the chronic spinal rat as compared to the normal adult rat.

Stimulation of the proximal and distal segments of the tail often resulted in inappropriate movements, i.e., movements directed toward the stimulus. This is clearly different from the situation in normal rats in which the percentage of correct movements is $\sim 100 \%$ (Fig. 2).

\section{Receptive fields of single hindlimb muscles}

In the decerebrate chronic spinal rat $(n=5)$, hindlimb reflexes were typically hyperexcitable, being evoked even by light touch. These reflex movements were often followed by movements irradiating to the tail and contralateral hindlimb. Because of the complex movements evoked by mechanical stimulation, nociceptive $\mathrm{CO}_{2}$ laser stimulation was used to map the receptive fields of the withdrawal reflexes. Periods of marked excitability fluctuations were sometimes observed; mapping was discontinued at such times. Reflexes were recorded in PER, EDL, and medial and lateral GA.

The well-ordered spatial organization of the receptive fields 
PER, and GA withdrawal reflexes in acute spinal rats (left column) and in rats whose spinal cords were transected at level T8-11 at birth (right column). $\mathrm{CO}_{2}$ laser stimulation was used to map the receptive fields. Light gray, dark gray, and black indicate areas of the skin from which the evoked responses were $0-30 \%, 30-70 \%$, and $70-100 \%$ of the maximal response, respectively. Number of rats included in each average is indicated below each receptive field.
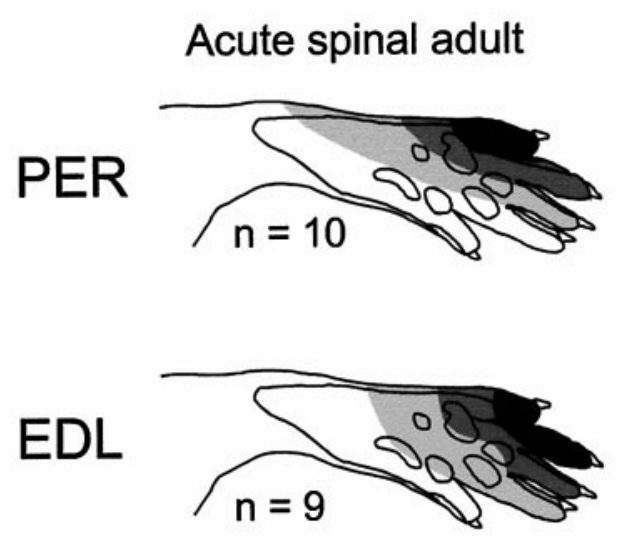

\section{Chronic spinal adult}

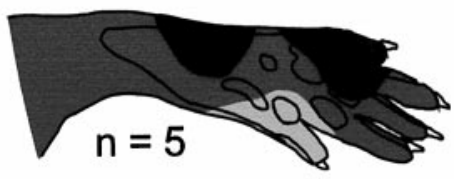

\section{GA}

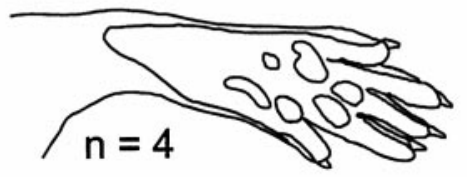

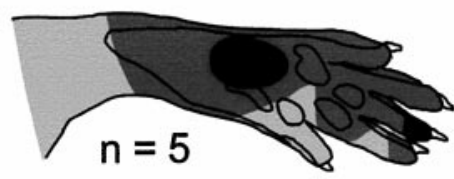

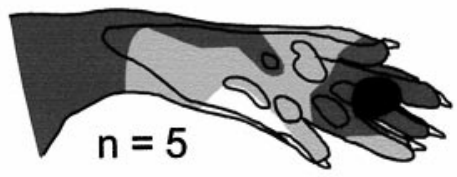

\section{Peroneus Longus}
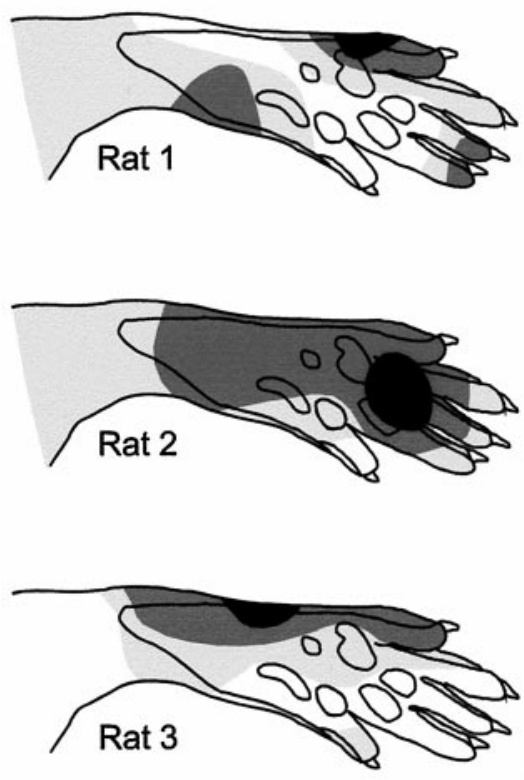

Figure 4. Samples of receptive fields of peroneus longus muscle obtained in single chronic spinal rats. Conventions as for Figure 3.

seen in acute spinal adult rats was disrupted in chronic spinal rats. Reflexes of PER could be elicited from the entire plantar side of the paw (Fig. 3, top row), with responses tending (on average) to be more prominent on the lateral side in the chronic spinal rat. The receptive field of this muscle clearly deviated the least from normal, but individual rats exhibited very abnormal receptive fields with irregularly located foci (Fig. 4). This has never been observed in normal adult rats.

In the chronic spinal rats, reflexes of EDL could be elicited from the entire plantar side of the paw (Fig. 3, middle row). Maximal responses were generally evoked from midplantar region. In normal rats, maximal responses in EDL are evoked from the distal part of the digits corresponding to the skin area maximally withdrawn on contraction in the muscle.

Reflexes of GA, which are abolished after acute spinalization in normal adult rats, were consistently evoked by $\mathrm{CO}_{2}$ laser stimulation in the chronic spinal rat (Fig. 3, bottom row). Responses could be evoked from almost the entire plantar surface, with maximal responses being evoked from different sites in different rats. The average receptive field showed a tendency for maximal responses to be evoked from the distal part of the foot. In the lightly anesthetized rat with intact spinal cord, maximal reflex responses in GA can be evoked from the heel, which corresponds to the maximally withdrawn skin area on muscle contraction (Schouenborg and Kalliomäki, 1990).

\section{Temporal characteristics of withdrawal reflexes}

Averaged poststimulus time histograms were generated to characterize the time course of reflex responses in each of the investigated muscles (Fig. 5). Data were sampled from the area evoking the maximal response in each chronic spinal rat. Data in acute spinal rats were sampled 4.5-6 hr after spinalization, i.e., after the spinal shock had worn off (Schouenborg et al., 1992), in the averaged focus area, which is also the focus area in each animal. The time courses of the $\mathrm{CO}_{2}$ laser-evoked reflexes were similar in acute and chronic spinal rats. The onset latency was $229 \pm 50$ and $200 \pm 47 \mathrm{msec}$ for acute and chronic spinal rats, respectively. As can be seen in Figure 5, the magnitudes of the responses evoked on stimulation of the aberrant foci of the chronic spinal rats and the normal foci of the acute spinal rats were approximately equal.

\section{DISCUSSION}

The present data on nocifensive behavior in awake rats and the receptive fields in decerebrate rats demonstrate that the postnatal tuning of the nociceptive withdrawal reflex system is markedly disturbed in the neonatally spinalized rat. Although the basic withdrawal reflex pathways are still present in the spinal cord, as suggested by a near normal time course of the reflex responses on thermal stimulation, the weight of the afferent input to individual modules is not functionally tuned. In addition, the relative reflex gain of plantar/dorsal flexors was found to be abnormal. These 

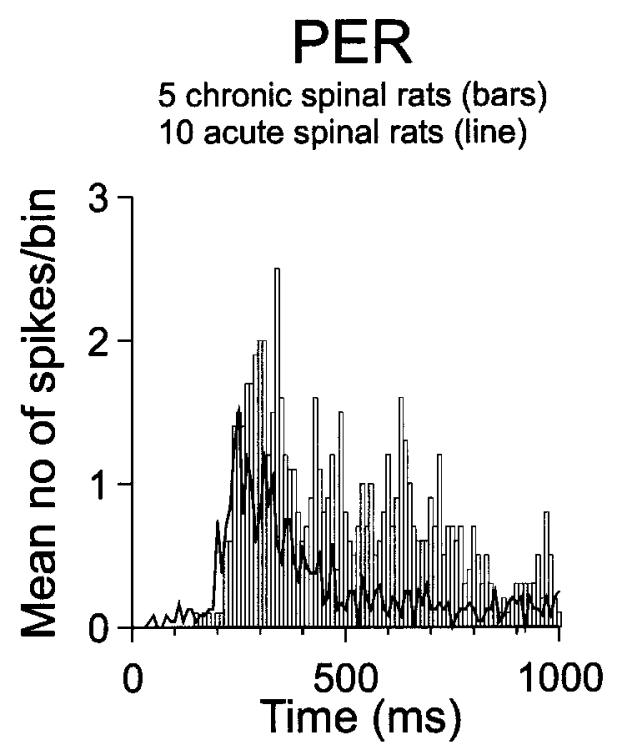

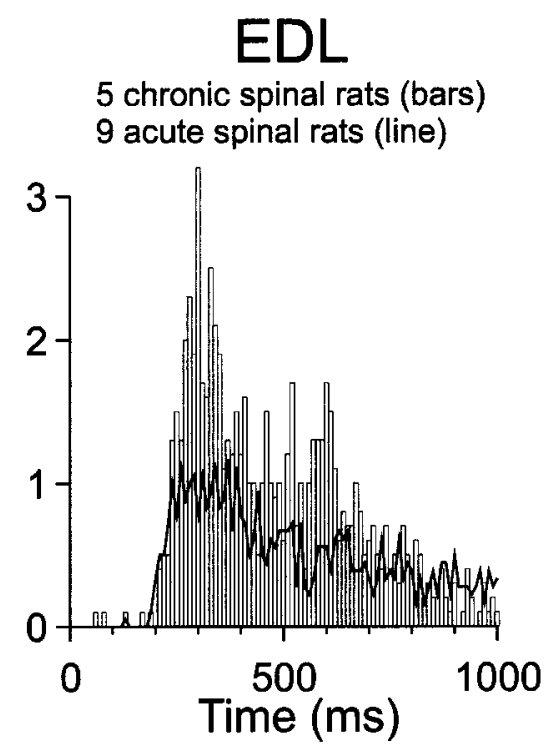

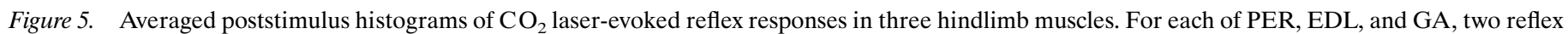

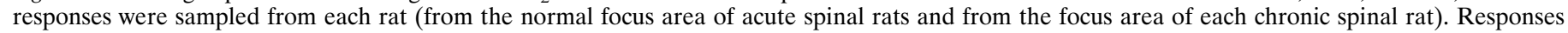

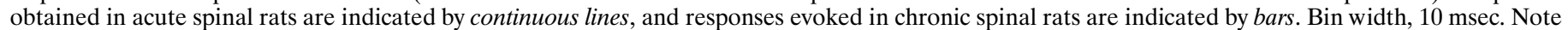
that the GA response is missing for acute spinal rats, because no reflexes can be evoked in this muscle in this preparation.

findings suggest a critical role for supraspinal systems in the postnatal tuning of spinal nociceptive systems.

\section{On the effects of spinalization in adult and neonatal rats}

In the adult rat, spinalization results in a short lasting "spinal shock", after which the receptive fields of both excitatory and inhibitory nociceptive reflex pathways expand, and the reflexes become hyperexcitable, reflecting a general loss of inhibitory control (Schouenborg et al., 1992; Weng and Schouenborg, 1996b). Importantly, this does not disrupt the sensitivity distribution within the receptive fields, at least not in the acute phase. Instead, the receptive fields expand along their gradients of sensitivity, with no displacement of foci, as the excitability increases. Excitability changes are known to occur also in humans after spinal lesions, although at a much slower pace, often leading to a severely debilitating hyperreflexia (Thilmann et al., 1991). The functional consequences of spinalization are far less severe in the neonatal than in the adult rat. Neonatally transected rats show little or no sign of spinal shock and develop stepping movements, placing reactions and extensor thrust reactions that have been reported to show similarities with normal responses (Stelzner, 1982; Miya et al., 1997). However, it is clear from the present data that the sensorimotor transformations are severely disturbed, at least in the withdrawal reflex system. Thus, supraspinal centers seem to be important not only for maintaining the integrity and proper excitability of adult spinal reflex circuits, but also for functionally tuning these circuits during postnatal development. The long-term consequences of spinalization on NWR function in adult rats are not known. However, maintenance of adequate reflex function may depend on continuous updating throughout life. Assuming that this requires supraspinal input, spinalization in the adult may eventually result in a complete disruption of the spatial organization of the NWRs.

\section{On the supraspinal control of spinal plasticity}

During the first three postnatal weeks, adequate sensorimotor transformations of the nociceptive withdrawal reflexes are at- tained. This is coincident with the maturation of descending inhibitory pathways in the dorsolateral funiculus (Fitzgerald and Koltzenburg, 1986). It may therefore be argued that the disturbed sensitivity distribution within the receptive fields of adult rats spinalized at birth results from a lack of general inhibitory influence from supraspinal centers. However, the emergence of the adult reflex organization, which presumably depends on experience-dependent mechanisms (Holmberg and Schouenborg, 1996a), reflects a selective upregulation and downregulation of adequate and erroneous connections, respectively. Therefore, a general lack of supraspinal inhibition cannot in itself explain the present findings.

It has previously been proposed that the tuning of NWR circuitry is initiated by activity in a reflex interneuron that triggers the motor neuron and thereby evokes a muscle twitch (Holmberg et al., 1997). The ensuing cutaneous sensory feedback would then be used to strengthen reflex connections from skin areas that are withdrawn, and/or weaken connections from skin receiving an increased load. In this context, it is of interest that long-term potentiation and depression of spinal synapses may play a role in the modulation of nociceptive transmission (Sandkuhler et al., 1997; Svendsen et al., 1997). These changes in synaptic gain have been shown to be critically dependent on the membrane potential of the postsynaptic spinal neuron (Randic et al., 1993), a phenomenon also described in the visual cortex (Artola et al., 1990) and the hippocampus (Stanton and Sejnowski, 1989). According to these studies, presynaptic activity in conjunction with a depolarized state of the postsynaptic neuron would favor potentiation, whereas a hyperpolarized state would favor depression of synaptic strength. If similar mechanisms were important in the tuning of NWRs, the lack of descending inhibition in the chronic spinal rat may result in a general tilt toward potentiation. This may prevent the elimination of erroneous connections to the nociceptive withdrawal reflex pathways.

From a series of studies on the visual, auditory, and sensorimotor cortices, it has been concluded that monoaminergic and cholinergic pathways play a "permissive" role for experience- 
dependent postnatal tuning (Singer, 1990; Baskerville et al., 1997; Kilgard and Merzenich, 1998; Kirkwood et al., 1999). The permissive role may, at least partly, be mediated through a general regulation of neuronal excitability. In view of the powerful descending control that monoaminergic and possibly cholinergic systems exert on nociceptive spinal systems (Bowker et al., 1983; Willis and Westlund, 1997), it is conceivable that similar mechanisms are also important for the postnatal tuning of the spinal nociceptive pathways.

\section{On the reflex gain in pathways to plantar flexors and dorsiflexors}

In the present study, brisk reflexes could be evoked in the gastrocnemius muscle in chronic spinal rats. Reflexes in this muscle have also been noted in acutely spinalized rats of up to 1 week of age (Holmberg and Schouenborg, 1996a), but never in acutely spinalized rats of 3 weeks of age or older. Furthermore, in the awake chronic spinal rat, reflex responses from noxious stimulation of the plantar side of the digits often incorporated plantar flexion, implicating a reflex response in plantar flexor muscles. Such responses elicited from the digits are never seen in the acutely spinalized adult rat or in the normal intact adult rat (Schouenborg et al., 1992; Holmberg and Schouenborg, 1996a). Thus, during early postnatal development, the reflex transmission for plantar flexors of the ankle and digits seems to be brought under a crucial descending control from supraspinal centers, where this descending influence is a requisite for normal transmission in the adult.

A related phenomenon may be the shift in balance between reflexes in dorsiflexors and plantar flexors, i.e., the "reversal" of the Babinski sign, in infants at $\sim 1$ year of age (van-Gijn, 1996). This change of reflex pattern is believed to reflect maturation of the corticospinal pathway (Porter and Lemon, 1993).

Indeed, several recent reports support the notion of a corticospinal control of spinal plasticity. For example, Wolpaw (1997) has demonstrated that the size of the monosynaptic Ia reflex can be altered if the reflex is associated with a reward. This plasticity does not occur if the corticospinal tract has been transected (Chen and Wolpaw, 1997). Furthermore, in normal human infants, the stretch reflexes are characterized by responses in both the stretched muscle and from the reciprocal or antagonistic muscle. The response in the antagonistic muscle is eliminated during normal development, but not in individuals with impaired descending influence, such as patients with cerebral palsy (Myklebust and Gottlieb, 1993).

\section{Implications for spinal cord injury and repair}

It is likely that a successful treatment for spinal cord injury will depend not only on regeneration of severed axons (Schwab, 1990; Davies et al., 1997; Tessler et al., 1997), but also on the capacity for reorganization of pre-existing spinal cord circuitry (Miya et al., 1997; Muir and Steeves, 1997). The present study shows that the circuitry underlying NWR is not tuned in the isolated spinal cord. Unraveling the mechanisms underlying the tuning of NWR and the role of descending pathways in this context could be an important contribution in the design of a strategy for using the reorganizational capacity of the spinal cord for the treatment of cord injuries.

\section{REFERENCES}

Artola A, Bröcher S, Singer W (1990) Different voltage-dependent thresholds for inducing long-term depression and long-term potentiation in slices of rat visual cortex. Nature 347:69-72.
Baskerville KA, Schweitzer JB, Herron P (1997) Effects of cholinergic depletion on experience-dependent plasticity in the cortex of the rat. Neuroscience 80:1159-1169.

Bowker RM, Westlund KN, Sullivan MC, Wilber JF, Coulter JD (1983) Descending serotonergic, peptidergic and cholinergic pathways from the raphe nuclei: a multiple transmitter complex. Brain Res 288:33-48.

Burke RE (1981) Motor units: anatomy and physiology, and functional organisation. In: Motor control, Handbook of physiology, Sect 1, The nervous system, Vol II, Part 1, (Brookhart JM, Mountcastle VB, Brooks VB, eds), pp 345-422. Baltimore: Williams \& Wilkins.

Chen XY, Wolpaw JR (1997) Dorsal column but not lateral column transection prevents down-conditioning of $\mathrm{H}$ reflex in rats. J Neurophysiol 78:1730-1734.

Clarke RW, Matthews B (1990) The thresholds of the jaw-opening reflex and trigeminal brainstem neurons to tooth-pulp stimulation in acutely and chronically prepared cats. Neuroscience 36:105-114.

Davies SJ, Fitch MT, Memberg SP, Hall AK, Raisman G, Silver J (1997) Regeneration of adult axons in white matter tracts of the central nervous system. Nature 390:680-683.

Fitzgerald M, Koltzenburg M (1986) The functional development of descending inhibitory pathways in the dorsolateral funiculus of the newborn rat spinal cord. Brain Res 389:261-270.

Holmberg H, Schouenborg J (1996a) Postnatal development of the nociceptive withdrawal reflexes in the rat: a behavioral and electromyographic study. J Physiol (Lond) 493:239-252.

Holmberg H, Schouenborg J (1996b) Developmental adaptation of withdrawal reflexes to early alteration of peripheral innervation in the rat. J Physiol (Lond) 495:399-409.

Holmberg H, Schouenborg J, Yu YB, Weng HR (1997) Developmental adaptation of rat nociceptive withdrawal reflexes after neonatal tendon transfer. J Neurosci 17:2071-2078.

Kilgard MP, Merzenich MM (1998) Cortical map reorganization enabled by nucleus basalis activity. Science $279: 1714-1718$.

Kirkwood A, Rozas C, Kirkwood J, Perez F, Bear MF (1999) Modulation of long-term synaptic depression in visual cortex by acetylcholine and norepinephrine. J Neurosci 19:1599-1609.

Kudo N, Furukawa F, Okado N (1993) Development of descending fibers to the rat embryonic spinal cord. Neurosci Res 16:131-141.

Leong SK, Shieh JY, Wong WC (1984) Localizing spinal-cordprojecting neurons in neonatal and immature albino rats. J Comp Neurol 228:18-23.

Mendell LM (1966) Physiological properties of unmyelinated fiber projection to the spinal cord. Exp Neurol 16:316-332.

Miya D, Giszter S, Mori F, Adipudi V, Tessler A, Murray M (1997) Fetal transplants alter the development of function after spinal cord transection in newborn rats. J Neurosci 17:4856-4872.

Muir GD, Steeves JD (1997) Sensorimotor stimulation to improve locomotor recovery after spinal cord injury. Trends Neurosci 20:72-77.

Myklebust BM, Gottlieb GL (1993) Development of the stretch reflex in the newborn: reciprocal excitation and reflex irradiation. Child Dev 64:1036-1045.

Porter R, Lemon R (1993) Corticospinal function and voluntary movement. Oxford: Oxford UP.

Randic M, Jiang MC, Cerne R (1993) Long-term potentiation and longterm depression of primary afferent neurotransmission in the rat spinal cord. J Neurosci 13:5228-5241.

Sandkuhler J, Chen JG, Cheng G, Randic M (1997) Low-frequency stimulation of afferent Adelta-fibers induces long-term depression at primary afferent synapses with substantia gelatinosa neurons in the rat. J Neurosci 17:6483-6491.

Schouenborg J (1984) Functional and topographical properties of field potentials evoked in rat dorsal horn by cutaneous C-fibre stimulation. J Physiol (Lond) 356:169-192.

Schouenborg J, Kalliomäki J (1990) Functional organization of the nociceptive withdrawal reflexes. I. Activation of hindlimb muscles in the rat. Exp Brain Res 83:67-78.

Schouenborg J, Weng HR (1994) Sensorimotor transformation in a spinal motor system. Exp Brain Res 100:170-174.

Schouenborg J, Holmberg H, Weng HR (1992) Functional organization of the nociceptive withdrawal reflexes. II. Changes of excitability and receptive fields after spinalization in the rat. Exp Brain Res 90:469-478.

Schouenborg J, Weng HR, Holmberg H (1994) Modular organization of spinal nociceptive reflexes. News Physiol Sci 9:261-265.

Schouenborg J, Weng HR, Kalliomäki J, Holmberg H (1995) A survey 
of spinal dorsal horn neurones encoding the spatial organization of withdrawal reflexes in the rat. Exp Brain Res 106:19-27.

Schouenborg J, Holmberg H, Luo XL, Levinsson A (1996) Adult rats spinalized at birth exhibit inappropriate withdrawal reflexes. Soc Neurosci Abstr 22:112.

Schwab ME (1990) Myelin-associated inhibitors of neurite growth and regeneration in the CNS. Trends Neurosci 13:452-456.

Singer W (1990) Ontogenetic self-organization and learning. In: Brain organization and learning (McGaugh JL, Weinberger NM, Lynch G, eds), pp 211-233. New York: Oxford Science.

Stanton PK, Sejnowski TJ (1989) Associative long-term depression in the hippocampus induced by Hebbian covariance. Nature 339:215-218.

Stelzner DJ (1982) The role of descending systems in maintaining intrinsic spinal function: a developmental approach. In: Brain stem control of spinal mechanisms (Sjölund B, Björklund A, eds), pp 297-321. Elsevier: Amsterdam.

Svendsen F, Tjolsen A, Hole K (1997) LTP of spinal A $\beta$ and C-fibre evoked responses after electrical sciatic nerve stimulation. NeuroReport 8:3427-3430.
Tessler A, Fischer I, Giszter S, Himes BT, Miya D, Mori F, Murray M (1997) Embryonic spinal cord transplants enhance locomotor performance in spinalized newborn rats. Adv Neurol 72:291-303.

Thilmann AF, Fellows SJ, Garms E (1991) The mechanism of spastic muscle hypertonus. Variation in reflex gain over the time course of spasticity. Brain 114:233-244.

van-Gijn J (1996) The Babinski sign: the first hundred years. J Neurol 243:675-683.

Weng HR, Schouenborg J (1996a) Cutaneous inhibitory receptive fields of withdrawal reflexes in the decerebrate spinal rat. J Physiol (Lond) 493:253-265.

Weng HR, Schouenborg J (1996b) Nociceptive inhibition of withdrawal reflex responses increases over time in spinalized rats. NeuroReport 7:1310-1314.

Willis WD, Westlund KN (1997) Neuroanatomy of the pain system and of the pathways that modulate pain. J Clin Neurophysiol 14:2-31.

Wolpaw JR (1997) The complex structure of a simple memory. Trends Neurosci 20:588-594. 